\title{
XENON1T solar axion and the Higgs boson emerging from the dark
}

\author{
Chengfeng Cai and Hong-Hao Zhang $\odot^{*}$ \\ School of Physics, Sun Yat-Sen University, Guangzhou 510275, China \\ Giacomo Cacciapaglia $^{\dagger}$ \\ Institut de Physique des 2 Infinis (IP2I), CNRS/IN2P3, UMR5822, 69622 Villeurbanne, France \\ and Université de Lyon, Université Claude Bernard Lyon 1, 69001 Lyon, France \\ Martin Rosenlyst $\odot^{\ddagger}$ and Mads T. Frandsen ${ }^{\S}$ \\ $C P^{3}$-Origins, University of Southern Denmark, Campusvej 55, DK-5230 Odense M, Denmark
}

(Received 15 July 2020; accepted 28 September 2020; published 15 October 2020)

\begin{abstract}
In a recent letter we proposed a new nonthermal mechanism of dark matter production based on vacuum misalignment, where both the Higgs boson and a very light pseudoscalar $\eta$ emerge from the dark sector. In this paper, we identify the parameter space in a composite scenario where the light pseudoscalar can be produced in the sun and explain the XENON1T excess in electron recoil data. The model's dark matter candidate has a mass around $50 \mathrm{TeV}$ and out of range for direct detection. Testable predictions include gravitational waves at frequencies in the $\mathrm{Hz}$ range from a cosmological phase transition, an exotic decay $Z \rightarrow \gamma+$ inv with rates $4 \div 16 \times 10^{-12}$ testable at a future Tera- $Z$ collider, and an enhancement by $17 \div 40 \%$ of the branching ratio $K_{L} \rightarrow \pi^{0}+$ inv, not enough to explain the KOTO anomaly. All these predictions may be confirmed by future experiments.
\end{abstract}

DOI: 10.1103/PhysRevD.102.075018

\section{INTODUCTION}

In a recent letter [1] we presented a novel mechanism that can produce the needed dark matter relic density in a nonthermal way. This framework applies when the electroweak symmetry breaking is due to vacuum misalignment and the Higgs boson emerges as a pseudo-NambuGoldstone boson (pNGB). A large class of models of new physics are thus eligible, ranging from models based on strong dynamics, like composite Higgs [2,3] and little Higgs [4,5] models, to weakly coupled models, like holographic extra dimensions [6,7] and elementary Goldstone boson models [8]. The kernel of the dark matter production mechanism lies in the thermal history of the cooling Universe: at high temperature, the vacuum of the model consists of an essentially Higgsless phase, where the electroweak symmetry is broken at a scale $f \gg v_{\mathrm{SM}}=246 \mathrm{GeV}$, and a global $\mathrm{U}(1)_{X}$ allows for the

\footnotetext{
*zhh98@mail.sysu.edu.cn

g.cacciapaglia@ipnl.in2p3.fr

rosenlyst@cp3.sdu.dk

§randsen@cp3.sdu.dk
}

Published by the American Physical Society under the terms of the Creative Commons Attribution 4.0 International license. Further distribution of this work must maintain attribution to the author(s) and the published article's title, journal citation, and DOI. Funded by SCOAP. generation of an asymmetric density of pNGBs deriving from a large global symmetry, spontaneously broken by the vacuum. At a temperature $T_{*}<f$, the vacuum starts rotating away from the Higgsless direction and toward an alignment where the Higgs boson can be identified with a light pNGB of the theory, while the $\mathrm{U}(1)_{X}$ is spontaneously broken. Part of the asymmetry is thus stored in stable $\mathbb{Z}_{2}$-odd pNGBs, the lightest of which plays the role of the dark matter currently filling in the Universe. In the Higgsless vacuum, the would-be Higgs boson $h$ forms a $\mathrm{U}(1)_{X}$ charged scalar, $\phi_{X}=(h+i \eta) / \sqrt{2}$, with a neutral pseudoscalar $\eta$. A generic prediction of the model is that $\eta$ remains very light in the zero-temperature vacuum. Thus, both the Higgs boson and a very light pseudoscalar emerge from the dark sector of the theory.

It is tantalizing that on June 17, 2020, the XENON1T collaboration unveiled the presence of an excess in the electron recoil events [9], which may be compatible with the production of an axionlike particle (ALP) in the sun. The mass of this new pseudoscalar should be below $100 \mathrm{eV}$ to explain the data. In this paper we will ask the question: can the pseudoscalar $\eta$, emerging from the dark, explain this excess?

While the dark matter production mechanism of Ref. [1] applies to a large variety of models, to study the XENON1T excess we will focus on the possibility that the dynamics is based on compositeness. The advantage of this approach is that the couplings of the $\eta$ to gauge bosons can be predicted 
in terms of the Wess-Zumino-Witten (WZW) topological anomaly $[10,11]$, while couplings to fermions are negligible in accordance to the $\mathrm{U}(1)_{X}$ symmetry. The minimal global symmetry breaking patterns that can accommodate the dark matter production mechanism are $\mathrm{SU}(6) / \mathrm{Sp}(6)$ and $\mathrm{SU}(4) \times \mathrm{SU}(4) / \mathrm{SU}(4) .{ }^{1} \mathrm{In}$ both cases, the WZW couplings of $\eta$ can be written as $[15,16]$

$$
\mathcal{L}_{\mathrm{WZW}}=\frac{C_{\mathrm{WZW}}}{\Lambda} \eta\left(g^{2} W_{\mu \nu}^{a} \tilde{W}^{a, \mu \nu}-g^{\prime 2} B_{\mu \nu} \tilde{B}^{\mu \nu}\right),
$$

where $W^{a}(B)$ indicate the gauge bosons of $\mathrm{SU}(2)_{L}\left(\mathrm{U}(1)_{Y}\right)$ with gauge coupling $g\left(g^{\prime}\right)$, and $\tilde{G}^{\mu \nu}=\frac{1}{2} \epsilon^{\mu \nu \rho \sigma} G_{\rho \sigma}$. The coefficient can be expressed as follows:

$$
\frac{C_{\mathrm{WZW}}}{\Lambda}=\frac{d_{\psi} \cos \theta}{64 \sqrt{2} \pi^{2} f}
$$

where the composite Higgs decay constant $f$ is related to the electroweak scale $v_{\mathrm{SM}}$ via the misalignment angle $\theta$, as $f \sin \theta=v_{\mathrm{SM}}$. Moreover, $d_{\psi}$ contains information about the underlying gauge dynamics leading to the formation of the composite pNGBs: namely, $d_{\psi}$ is the dimension of the representation of the fermions $\psi$, which form the pNGBs, under the confining hypercolor gauge symmetry $\mathcal{G}_{\mathrm{HC}}$. For the coset $\mathrm{SU}(6) / \mathrm{Sp}(6)$, the minimal model is based on $\mathcal{G}_{\mathrm{HC}}=$ $\mathrm{SU}(2)_{\mathrm{HC}}$ with $\psi$ in the fundamental $\left(d_{\psi}=2\right)[15,17]$, while top partial compositeness can be obtained for $\mathcal{G}_{\mathrm{HC}}=$ $\mathrm{Sp}(4)_{\mathrm{HC}}\left(d_{\psi}=4\right) \quad[18,19]$. For $\mathrm{SU}(4) \times \mathrm{SU}(4) / \mathrm{SU}(4)$, the minimal model is based on $\mathcal{G}_{\mathrm{HC}}=\mathrm{SU}(3)_{\mathrm{HC}}$ with $\psi$ in the fundamental $\left(d_{\psi}=3\right)$ [16,20], while top partial compositeness can be generated for $\mathcal{G}_{\mathrm{HC}}=\mathrm{SU}(4)_{\mathrm{HC}}$ with $\psi$ in the fundamental $\left(d_{\psi}=4\right)[19,21]$. In the following, we will prefer models with top partial compositeness, and fix $d_{\psi}=4$ as the minimal choice. Once the underlying dynamics is fixed, the interactions of $\eta$ only depends on $f,{ }^{2}$ as $\cos \theta \sim 1$ due to precision bounds [22,23]. The other remaining free parameter is the mass. The system is, therefore, very constrained and the XENON1T excess offers a golden chance to test it against data.

\section{THE MODEL IN THE XENON1T ARENA}

At energies well below the electroweak scale, the $\eta$ couplings match those of a generic ALP [24]:

$$
\begin{aligned}
& \mathcal{L}_{\eta} \supset \frac{\partial^{\mu} \eta}{2} \sum_{\mathrm{f}} \frac{C_{\mathrm{ff}}}{\Lambda} \bar{\psi}_{\mathrm{f}} \gamma_{\mu} \gamma^{5} \psi_{\mathrm{f}}+e^{2} \frac{C_{\gamma \gamma}}{\Lambda} \eta F_{\mu \nu} \tilde{F}^{\mu \nu} \\
&+\frac{2 e^{2}}{s_{W} c_{W}} \frac{C_{\gamma Z}}{\Lambda} \eta F_{\mu \nu} \tilde{Z}^{\mu \nu}+\cdots
\end{aligned}
$$

\footnotetext{
${ }^{1}$ In both cases, the $\mathbb{Z}_{2}$ stable states may also be standard freezeout thermal candidates [12], see [13,14].

${ }^{2}$ Changing $d_{\psi}$ can be absorbed in a simple rescaling of $f$, as evident in Eq. (2).
}

where the dots contain couplings to $W^{+} W^{-}$and $Z Z$, which are irrelevant here. Of the above couplings, the WZW term of Eq. (1) only generates a nonvanishing

$$
\frac{C_{\gamma Z}}{\Lambda}=\frac{C_{\mathrm{wZW}}}{\Lambda} .
$$

Instead, $C_{\gamma \gamma}$ and $C_{\mathrm{ff}}$ are generated at loop level, and we refer the reader to Ref. [24] for all the necessary details. For the fermions, the loops are divergent and we will assume that they are cancelled by a counterterm at a scale close to the compositeness one, $\mu=f$. Thus, the physical couplings can be written as

$$
\begin{aligned}
\frac{C_{\mathrm{ff}}^{1-\text { loop }}(\mu)}{\Lambda}= & -\frac{3 \alpha^{2}\left(m_{Z}\right)}{2} \frac{C_{\mathrm{WZW}}}{\Lambda} \ln \frac{\mu^{2}}{m_{W}^{2}} \\
& \times\left(\frac{3}{s_{W}^{4}}-\frac{4}{c_{W}^{4}}\left(Y_{\mathrm{f}_{L}}^{2}+Y_{\mathrm{f}_{R}}^{2}\right)\right),
\end{aligned}
$$

where $\mathrm{f}$ indicates any fermion in the Standard Model, and $Y_{\mathrm{f}_{L / R}}$ are the hypercharges of the left and right-handed fields. Numerically, for $f=50 \mathrm{TeV}$ (and $d_{\psi}=4$ ), we find:

$$
\begin{gathered}
\frac{C_{\gamma \mathrm{Z}}}{\Lambda}=0.9 \times 10^{-7} \mathrm{GeV}^{-1}, \\
\frac{C_{e e}}{\Lambda}=-0.55 \times 10^{-8} \mathrm{GeV}^{-1}, \\
\frac{C_{u u}}{\Lambda}=-0.60 \times 10^{-8} \mathrm{GeV}^{-1}, \\
\frac{C_{d d}}{\Lambda}=-0.63 \times 10^{-8} \mathrm{GeV}^{-1} .
\end{gathered}
$$

The scaling with $f$ can be easily inferred from Eqs. (2) and (5). In our benchmark point, the axion coupling $g_{a e}$, relevant for the XENON1T signal, is given by:

$$
g_{a e}=\frac{m_{e} C_{e e}}{\Lambda}=-2.80 \times 10^{-12} .
$$

As the couplings to up and down are approximately equal, the effective coupling to nucleons receives a dominant contribution from the isosinglet coupling, thus

$$
g_{a n}^{\mathrm{eff}} \approx 0.36 \frac{m_{n}}{\Lambda} \frac{C_{u u}+C_{d d}}{2}=-2.08 \times 10^{-9}
$$

The coupling to photons, $C_{\gamma \gamma}$, is also generated by loops, and it receives competing contributions both at one-loop from the $W$, and at two-loop from the fermions. Its expression contains several contributions, and we refer the reader to Ref. [24] for more details. As we are interested 
in $\eta$ masses below $100 \mathrm{eV}$, which is below the $e^{+} e^{-}$ threshold, we find that $C_{\gamma \gamma}$ is suppressed by $m_{\eta}^{2}$, thus it is very small. In our benchmark, we find

$$
g_{a \gamma}=\frac{C_{\gamma \gamma}}{\Lambda} \approx-10^{-19} \mathrm{GeV}^{-1}\left(\frac{m_{\eta}}{100 \mathrm{eV}}\right)^{2}
$$

which is well below the sensitivity of XENON1T or of any other experiment. This small value also allows us to avoid astrophysical and cosmological bounds associated to photon couplings [24].

The XENON1T collaboration has presented the fit to a generic solar axion model in the parameter space $\left(\left|g_{a e}\right|\right.$, $\left.\left|g_{a e} g_{a n}^{\text {eff }}\right|,\left|g_{a e} g_{a \gamma}\right|\right)$, providing projections of the allowed pseudocuboid on the 3 surfaces. For our model, we can effectively consider $g_{a \gamma}=0$. This provides the following preferred ranges at $90 \%$ C.L.:

$$
\begin{gathered}
2.6 \times 10^{-12}<\left|g_{a e}\right|<3.7 \times 10^{-12} \\
\left|g_{a e} g_{a n}^{\mathrm{eff}}\right|<4.5 \times 10^{-18}
\end{gathered}
$$

for $m_{\eta}<100 \mathrm{eV}$. These boundaries define the blue region in Fig. 1. In the plot, we also report in black the excluded regions by other direct experiments: solar neutrino probes, LUX and PandaX-II. The green band represents the $2 \sigma$ region that would fit the observed anomalous cooling of stars [25,26], as reported by the XENON1T collaboration. We will come back to this tension toward the end of this section.

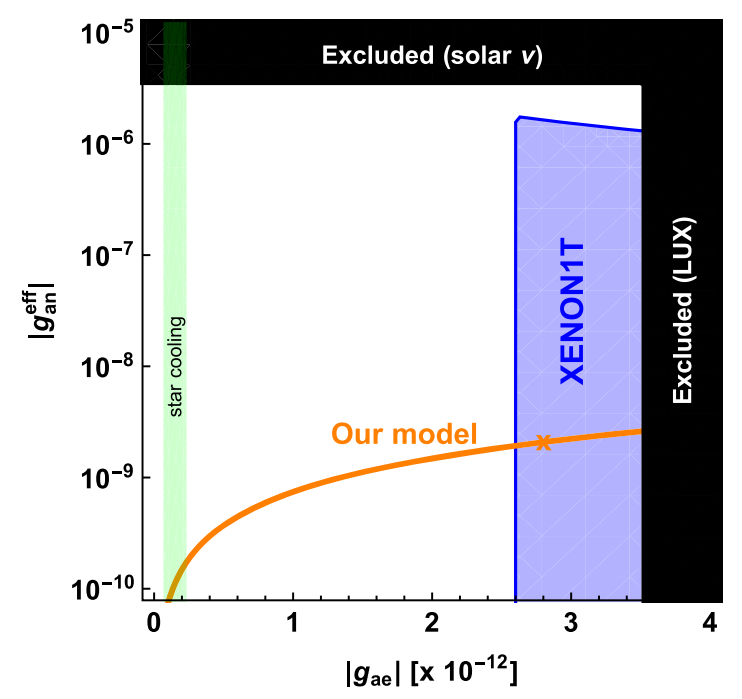

FIG. 1. In blue, 90\% C.L. region preferred by the XENON1T excess [9] under the solar axion hypothesis, compared to the prediction of our model in orange. Here we assume $g_{a \gamma} \approx 0$. The black area indicates the excluded are from other direct probes, while the green band could fit the anomalous cooling of stars [25]. The orange " $x$ " indicates the benchmark point $f=50 \mathrm{TeV}$.
The orange line in the plot shows the predictions for the pseudoscalar $\eta$ in our model. The shape of the line can be understood in terms of the ratio:

$$
\left|\frac{g_{a n}^{\mathrm{eff}}}{g_{a e}}\right|=743
$$

which is independent on $f$. The points inside the 90\% C.L. XENON1T region correspond to

$$
36<\frac{f}{\mathrm{TeV}}<55
$$

As $f$ is the decay constant of the composite pNGB Higgs, these large values point toward a very fine tuned situation at the electroweak scale [27]:

$$
\Delta_{\text {f.t. }}=\frac{v_{\mathrm{SM}}^{2}}{f^{2}}=2 \div 5 \times 10^{-5}
$$

In other words, the pNGB Higgs potential at zero temperature needs to be fine-tuned at the level of a part in $10^{5}$. While not optimal, this is still a solution to the hierarchy problem between the Planck and electroweak scales: without any new physics, a tuning at the level of one part in $10^{30}$ would be needed to keep the Higgs scale low. As we will discuss in the next section, fixing $f$ to reproduce the XENON1T excess in our model gives interesting predictions about other phenomena, which could be observed in the future. Of course, the first test of this model, as well as of any other explanation, will be in the data taken by the upgraded XENONnT detector [28] and LZ [29], which will be able to confirm, or rule as a mere statistical fluctuation, the excess.

The observed excess could also derive for an unexpected background, as discussed in the XENON1T paper [9]: adding tritium contamination has the effect of reducing the statistical significance for the solar axion from $3.6 \sigma$ to $2 \sigma$ (see also Ref. [30]). Astrophysical bounds from stellar cooling are at odds with the preferred region, as already pointed out by the XENON1T collaboration, message strengthened in Ref. [31]. The main point is that values of the coupling $g_{a e} \sim \mathcal{O}\left(10^{-12}\right)$ would cool down various types of stars too quickly. The observable that gives the strongest constraint is the ratio $R$ between the number of stars in two populations (horizontal branch over red giants branch) in globular clusters, which has been experimentally measured with a precision of a few percent. The tension has been evaluated to above $19 \sigma$ in Ref. [31]. The main mechanism behind this tension is the cooling of red giant cores due to the emission of axions, which leads to starts in the horizontal branch with a much more massive helium core and a shorter lifetime. However, the effect of the axion couplings on the star evolutions is estimated by interpolating the outcome of various stellar evolution 
simulations [26,32], where no theoretical error is provided. The cooling due to axions depends on many parameters of the star, which are not accurately known. As an example, we recall that the primary process of interest here, electron bremsstrahlung, is very sensitive to the temperature of the core of the star, which is not directly measurable. For these reasons, we pragmatically take star cooling bounds with a grain of salt, unless theoretical errors on the simulations and stellar models are properly propagated on the final results. We thus stand with the attitude of the XENON1T collaboration in considering the solar axion hypothesis is still valid. Note also that the inclusion of the inverse Primakoff effect at the detector level, as pointed out in Ref. [33], also reduces the tension, however only in models with large coupling to photon, unlike ours.

\section{PREDICTIONS}

The beauty of the light pseudoscalar $\eta$ emerging from the dark, in composite scenarios, is that its couplings to all Standard Model particles are ruled by a single parameter: the composite Higgs decay constant $f$. Once it is fixed to reproduce the XENON1T excess, we can predict several phenomena that may be observable in the near future and confirm, or rule out, this model.

\section{A. Dark matter and gravitational waves}

As the $\eta$ particle emerges from a nonthermal production mechanism for the dark matter, we will start with discussing this aspect of the theory. To do so, we have repeated the analysis presented in [1] for the benchmark point $f=50 \mathrm{TeV}$, as shown in Fig. 2 (which corresponds to the figure in [1], to which we refer the reader for details). The dynamics of the dark matter evolution depends on two

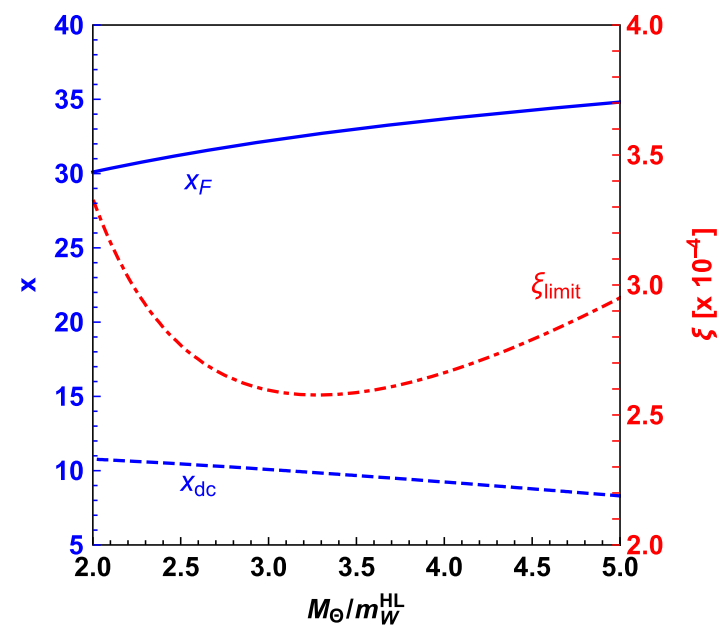

FIG. 2. In blue, the freeze-out $x_{F}$ and decoupling $x_{d c}$ parameters for the $\mathbb{Z}_{2}$-odd states as a function of $M_{\Theta} / m_{W}^{H L}$ for $m_{W}^{\mathrm{HL}}=$ $16 \mathrm{TeV}(f=50 \mathrm{TeV})$. In red, the value of coupling $\xi$ that provides the observed relic density from the asymmetry (smaller values are excluded). crucial temperatures: (a) the thermal freeze-out temperature $T_{F}=M_{\Theta} / x_{F}$, where $M_{\Theta}$ is the mass of the dark states in the high-temperature Higgsless vacuum, and (b) the decoupling temperature $T_{d c}=M_{\Theta} / x_{d c}$ where the processes transferring the asymmetry between the $\mathbb{Z}_{2}$-even and $\mathbb{Z}_{2}$-odd sectors decouple. The latter is important in fixing the relic abundance of dark matter, originating from the asymmetry, while $T_{F}$ is the largest temperature where the system can start drifting away from the Higgsless vacuum alignment, and toward the zero-temperature one (corresponding to the Standard Model). The values found in Fig. 2 demonstrate that the values for $f$ preferred by the XENON1T excess can provide a viable DM candidate.

In the Higgsless vacuum, the $W$ mass is thus given by $m_{W}^{\mathrm{HL}}=m_{W} f / v_{\mathrm{SM}}=16 \mathrm{TeV}$, while $M_{\Theta}$ is the mass of the $\mathbb{Z}_{2}$-odd states. One important parameter that fixes the correct relic density is the coupling $\xi$, which at zero temperature corresponds to the coupling between the dark matter and the Higgs boson: the preferred values $\xi \approx$ few $\times 10^{-4}$ imply that direct detection of the $M_{\mathrm{DM}} \approx M_{\Theta} \approx$ $50 \mathrm{TeV}$ candidate is out of reach.

The dark matter production mechanism also requires that the theory undergoes a strong order phase transition, where the strong dynamics confines, at temperatures $T_{\mathrm{HL}} \approx f$. This phase transition may generate gravitational waves [34]. The peak frequency of the diffuse gravitational waves mainly depends on the temperature of the phase transition: for instance, from Ref. [35] we can estimate the peak frequency

$$
\omega_{\text {peak }} \approx 1 \mathrm{~Hz} \frac{1}{v_{\mathrm{w}}}\left(\frac{\beta / H_{*}}{1000}\right)\left(\frac{T_{\mathrm{HL}}}{100 \mathrm{TeV}}\right)
$$

where $\beta / H_{*} \approx \mathcal{O}(100)$ is a parameter related to the bubble formation, and $v_{\mathrm{w}} \approx 1$ is the wall velocity. We thus expect the peak frequency to be in the Hz ballpark, which matches the sensitivity of future detection experiments like Voyager, the Einstein Telescope and the Big Bang Observer (BBO). We leave a more detailed analysis of the gravitational wave spectrum and amplitude for future work.

\section{B. Future $e^{+} e^{-}$collider signals}

The coupling $C_{\gamma Z}$ in Eq. (4) induces decays of the $Z \rightarrow \gamma \eta$, where $\eta$ will appear as missing energy in a detector due to the long lifetime. In fact, for masses in the XENON1T interesting range, $m_{\eta}<100 \mathrm{eV}$, the dominant decay mode is in neutrinos (as the coupling to photons is negligible), which is suppressed by the neutrino masses and always gives a cosmological stable $\eta$. This exotic decay of the $Z$ could be detectable at a future $e^{+} e^{-}$collider running at the $Z$ pole, providing a final state with a single monoenergetic photon and missing energy. The branching ratio is given by 


$$
\Gamma(Z \rightarrow \gamma \eta)=\frac{8 \pi \alpha^{2}\left(m_{Z}\right)}{3 s_{W}^{2} c_{W}^{2}}\left(\frac{C_{\gamma Z}}{\Lambda}\right)^{2} m_{Z}^{3}
$$

which gives the following prediction in the range for $f$ preferred by the XENON1T excess:

$$
6<\frac{\operatorname{BR}(Z \rightarrow \gamma \eta)}{10^{-12}}<14
$$

For a future $e^{+} e^{-}$collider, a Tera- $Z$ run would provide between 6 and 14 events, which may be detectable over the small standard model background. The analysis would be very simple, and it has already been performed at LEP [36-38], where a bound $\operatorname{BR}(Z \rightarrow \gamma+$ inv $)<10^{-6}$ was established.

Future plans also include the possibility of a high-energy hadron collider, to be built either in Europe at CERN or in China, which could reach center-of-mass energies of $100 \mathrm{TeV}$. However, resonances produced by the composite dynamics, having a typical mass much larger than $f$, will be too heavy to be directly produced. Thus, the only hope remains for additional pNGBs rather than the Higgs and the ALP $\eta$. In the template model we consider here, all the additional pNGBs are odd, and heavier than the DM states. As they need to be pair produced, a $100 \mathrm{TeV}$ collider will not have sufficient energy.

\section{Flavor changing decays of $\boldsymbol{K}$ and $\boldsymbol{B}$ mesons}

The loop-induced couplings of the light pseudoscalar $\eta$ in Eq. (5) have been computed by extracting the divergent piece of the loop, which is independent on the mass of the quark in the loop. This is a good approximation for loops containing fermions much lighter than the $W$ and $Z$ masses, i.e., for all Standard Model fermions except the top quark. This point has an important consequence: $W$-loops with the top quark can generate flavor off-diagonal contribution to the coupling of $\eta$ to down-type quarks. Parametrizing them in analogy to Eq. (3) as

$$
\mathcal{L}_{\eta} \supset \frac{\partial^{\mu} \eta}{2} \sum_{i \neq j} \frac{C_{d^{i} d^{j}}}{\Lambda} \bar{\psi}_{d^{i}} \gamma_{\mu} \gamma^{5} \psi_{d^{j}}
$$

an explicit calculation yields

$$
\frac{C_{d^{i} d^{j}}}{\Lambda}=-\frac{3 \alpha^{2}\left(m_{Z}\right)}{s_{W}^{4}} \frac{C_{W Z W}}{\Lambda} V_{t d^{i}}^{*} V_{t d^{j}} \frac{1-r_{W}-\ln r_{W}}{\left(1-r_{W}\right)^{2}}
$$

where $V_{t d^{i}}$ are entries of the CKM matrix and $r_{W}=m_{W}^{2} / m_{t}^{2}$. Interestingly, sizeable off-diagonal couplings only arise in the down quark sector. For the benchmark point $f=50 \mathrm{TeV}$, we find

$$
\frac{C_{s d}}{\Lambda}=-3.3 \times 10^{-13} \mathrm{GeV}^{-1}
$$

$$
\begin{aligned}
& \frac{C_{b s}}{\Lambda}=-4.8 \times 10^{-11} \mathrm{GeV}^{-1}, \\
& \frac{C_{b d}}{\Lambda}=-1.0 \times 10^{-11} \mathrm{GeV}^{-1},
\end{aligned}
$$

where we only report the real part of the coupling.

The first coupling between strange and down quarks is of particular interest, as it can mediate the decay of $K \rightarrow \pi+$ inv, where the invisible momentum is carried away by the $\eta$ pseudoscalar. This process for neutral Kaons has been recently measured by the KOTO experiment [39], which has reported a significant excess over the Standard Model prediction [40]:

$$
\begin{gathered}
\left.\mathrm{BR}\left(K_{L} \rightarrow \pi^{0}+\text { inv }\right)\right|_{\text {кото }}=2.1_{-1.7}^{+4.1} \times 10^{-9}, \\
\left.\mathrm{BR}\left(K_{L} \rightarrow \pi^{0}+\text { inv }\right)\right|_{\mathrm{SM}}=3.0 \pm 0.3 \times 10^{-11},
\end{gathered}
$$

with errors at $90 \%$ C.L.. To explain this large enhancement with respect to the Standard Model prediction is at odds with the measurement of the decay of the charged Kaon from NA62 experiment

$$
\begin{gathered}
\left.\operatorname{BR}\left(K^{+} \rightarrow \pi^{+}+\text {inv }\right)\right|_{\text {NA62 }}<2.44 \times 10^{-10}, \\
\left.\operatorname{BR}\left(K^{+} \rightarrow \pi^{+}+\text {inv }\right)\right|_{\mathrm{SM}}=9.11 \pm 0.72 \times 10^{-11},
\end{gathered}
$$

as given by the Grossman-Nir bound [41].

In our model, the calculation can be performed following the same procedure as in Ref. [42]: we find

$$
\begin{aligned}
\Gamma_{K \rightarrow \pi+\mathrm{inv}}= & \frac{\lambda^{1 / 2}\left(1, \hat{m}_{\pi}^{2}, \hat{m}_{\eta}^{2}\right) m_{K}^{3}}{64 \pi}\left(\frac{C_{s d}}{\Lambda}\right)^{2} \\
& \times\left[\left(1-\hat{m}_{\pi}^{2}\right) f_{+}+\hat{m}_{\eta}^{2} f_{-}\right],
\end{aligned}
$$

where $f_{+} \approx 1$ and $f_{-} \approx-0.28$ are form factors, $\lambda(a, b, c)=a^{2}+b^{2}+c^{2}-2 a b-2 a c-2 b c$ is the usual kinematical function and $\hat{m}_{X}=m_{X} / m_{K}$. This formula applies both to $K^{+}$and to $K_{L}$, so the difference in branching ratios will only come from the different lifetimes of the Kaon, thus respecting the Grossman-Nir bound. For the XENON1T preferred region, we find

$$
\begin{gathered}
\operatorname{BR}\left(K_{L} \rightarrow \pi^{0}+\text { inv }\right)=0.5 \div 1.2 \times 10^{-11}, \\
\operatorname{BR}\left(K^{+} \rightarrow \pi^{+}+\text {inv }\right)=0.12 \div 0.29 \times 10^{-11}
\end{gathered}
$$

For $K_{L}$, the new physics channel enhances the Standard Model prediction by $17 \%$ to $40 \%$, which is not enough to explain the KOTO anomaly. Yet, if the precision reaches the level of the Standard Model, and the KOTO anomaly is not confirmed, such enhancement may be a confirmation of the model. For the charged Kaon, the effect is much smaller, at the percent level. 
The couplings involving the bottom quark induce similar decays for the $\mathrm{B}$ mesons into pions (via $C_{b d}$ ) and Kaons (via $C_{b s}$ ). A calculation similar to the one done for the Kaons, leads to the predictions

$$
\begin{aligned}
& \operatorname{BR}(B \rightarrow K+\text { inv })=3.4 \div 8.0 \times 10^{-9}, \\
& \operatorname{BR}(B \rightarrow \pi+\text { inv })=1.5 \div 3.4 \times 10^{-10},
\end{aligned}
$$

roughly the same for charged and neutral mesons due to the similar lifetimes. The experimental bounds on all those processes are at the level of $10^{-5}$ [43-45], while the Standard Model predictions read [46-48]

$$
\begin{gathered}
\mathrm{BR}(B \rightarrow K \nu \nu)=4.0 \pm 0.5 \times 10^{-6}, \\
\mathrm{BR}\left(B^{+} \rightarrow \pi^{+} \nu \nu\right)=2.4 \pm 0.3 \times 10^{-7}, \\
\mathrm{BR}\left(B^{0} \rightarrow \pi^{0} \nu \nu\right)=1.2 \pm 0.15 \times 10^{-7} .
\end{gathered}
$$

The correction deriving from our model, therefore, is undetectable in the $\mathrm{B}$ meson sector.

\section{DISCUSSION}

The observation of an excess in the electron recoil spectrum for energies below a few $\mathrm{keV}$ by the XENON1T has open the tantalising possibility that some kind of light new physics have been observed. The explanation that best fits the data is that of an axion of solar origin, with masses below $100 \mathrm{eV}$. The inclusion of an unknown Tritium background, can however reduce the evidence from $3.6 \sigma$ to about $2 \sigma$, therefore further data are necessary to confirm this excess as a genuine source of new physics.

The fact that the solar axion hypothesis seems at odds with solar cooling models [31], has ignited the community in finding alternative explanations: for instance, the signal could be due to a photophobic warm dark matter axion [49] or another boson produced in the sun [50-53]. The possibility of exotic solar neutrino interactions, induced by a light mediator, has also been considered by several authors [54-59], as well as a variety of mechanism that could boost a light dark matter candidate [52,58,60-68], with some consensus around models with two states split by only a few $\mathrm{keV}$. Some cases consider decays of mesons [69] or the hydrogen atom [70] to the dark sector. Finally, the possibility of a non-thermal hidden photon dark matter [71-73], of photons emitted by dark matter [74-76], mirror symmetry [77], U(1)-2HDM [78] or SIMP dark matter models [79] have been considered.

In this paper, we stand for the solar axion explanation that offers the best fit to the data. The estimates for the axion production in stars suffer from intrinsic uncertainties, thus the deriving bounds should be taken with a grain of salt. We find that a good candidate was predicted by a new mechanism of dark matter production we proposed in
Ref. [1], in a composite Higgs scenario. Besides the mass, which is expected to be very light and can be in the range relevant for the XENON1T excess, $m_{\eta}<100 \mathrm{eV}$, the couplings of the ALP $\eta$ to Standard Model particles are all ruled by a single parameter: the Higgs decay constant $f$. Once $f$ is fixed in the range $36-55 \mathrm{TeV}$ to explain the XENON1T excess, the model issues several predictions that are testable at future experiments.

First, the large value of the Higgs decay constant implies a severe fine tuning in the Higgs potential, of the order of a few parts in $10^{5}$ : while still being a solution to the hierarchy problem with the Planck scale, this implies that the LHC cannot probe the composite nature of the Higgs, while a $100 \mathrm{TeV}$ hadron collider may be barely sufficient. On the other hand, the lightness of $\eta$ implies the exotic decay $Z \rightarrow \gamma+$ inv, with rates between $6 \div 14 \times 10^{-12}$. A future Tera-Z run of the $e^{+} e^{-}$collider, as recommended by the European Strategy 2020, would have a handful of events, which may be observable due to the very low background and the monochromatic nature of the photon.

Furthermore, the dark matter candidate is predicted to have masses in the $50 \mathrm{TeV}$ range and small couplings to the Higgs boson, which makes its direct detection out of reach. On the bright side, the necessity for a strong first order phase transition at a temperature close to $f$ implies the production of gravitational waves with a typical peak frequency around the $\mathrm{Hz}$, thus being potentially detectable by future experiments like Voyager, the Einstein Telescope and the Big Bang Observer (BBO).

The light pseudoscalar also implies exotic decays of the Kaons, $K_{L} \rightarrow \pi^{0}+$ inv, with sizeable rates. This is due to flavor changing couplings of the ALP to down-type quarks, generated by top loops. The fact that such effects are only expected for down-type quarks is a nice consequence of the loop nature of these couplings. We predict that the rate $K_{L} \rightarrow \pi^{0}+$ inv receives a $+17 \div 40 \%$ enhancement due to the new physics channel. This is not enough to explain the recent KOTO anomaly, but it could be detected once experimental precision reaches the level of the Standard Model prediction. Other channels, like $K^{+} \rightarrow \pi^{+}+$inv, $B_{0} \rightarrow K_{0} / \pi^{0}+$ inv and $B^{+} \rightarrow K^{+} / \pi^{+}+$inv, all receive much smaller contributions.

In conclusion, we have shown that composite models with the Higgs boson emerging from the dark predict a very light pseudo-scalar, which may explain the XENON1T excess as a solar axion, with the bonus of testable prediction in various phenomena ranging from gravitational waves to Kaon physics.

\section{ACKNOWLEDGMENTS}

G. C. acknowledges partial support from the Labex-LIO (Lyon Institute of Origins) under Grant No. ANR-10LABX-66 (Agence Nationale pour la Recherche), and 
FRAMA (FR3127, Fédération de Recherche "André Marie Ampère"). C. C. and H. H. Z. are supported by the National Natural Science Foundation of China (NSFC) under Grants No. 11875327 and No. 11905300, the China Postdoctoral Science Foundation under Grant No. 2018M643282, the Natural Science Foundation of Guangdong Province under Grant No. 2016A030313313, the Fundamental Research Funds for the Central Universities, and the Sun Yat-Sen University Science Foundation. M. T. F. and M. R. acknowledge partial funding from The Council For Independent Research, Grant No. DFF 6108-00623. The CP3-Origins centre is partially funded by the Danish National Research Foundation, Grant No. DNRF90. G. C., C. C., and H. H. Z. also acknowledge support from the China-France Laboratoire International Associé (LIA) France-China Particle Physics Laboratory (FCPPL). G. C. also thanks the Sun Yat-Sen University for hospitality during the completion of this project.

Note added.-After the completion of this work, Ref. [80] presented an analysis of the decay of the $Z$ boson to a single photon plus missing energy, similar to the signal predicted here. The authors considered decays into a dark photon instead of a pseudoscalar, finding that the FCC-ee could reach down to branching ratios of $10^{-11}$.
[1] C. Cai, H.-H. Zhang, G. Cacciapaglia, M. T. Frandsen, and M. Rosenlyst, Higgs Boson Emerging from the Dark, Phys. Rev. Lett. 125, 021801 (2020).

[2] D. B. Kaplan and H. Georgi, $\mathrm{SU}(2) \times \mathrm{U}(1)$ breaking by vacuum misalignment, Phys. Lett. 136B, 183 (1984).

[3] M. J. Dugan, H. Georgi, and D. B. Kaplan, Anatomy of a composite Higgs model, Nucl. Phys. B254, 299 (1985).

[4] N. Arkani-Hamed, A. G. Cohen, and H. Georgi, Electroweak symmetry breaking from dimensional deconstruction, Phys. Lett. B 513, 232 (2001).

[5] N. Arkani-Hamed, A. G. Cohen, E. Katz, A. E. Nelson, T. Gregoire, and J. G. Wacker, The minimal moose for a little Higgs, J. High Energy Phys. 08 (2002) 021.

[6] R. Contino, Y. Nomura, and A. Pomarol, Higgs as a holographic pseudoGoldstone boson, Nucl. Phys. B671, 148 (2003).

[7] Y. Hosotani and M. Mabe, Higgs boson mass and electroweak-gravity hierarchy from dynamical gauge-Higgs unification in the warped spacetime, Phys. Lett. B 615, 257 (2005).

[8] T. Alanne, H. Gertov, F. Sannino, and K. Tuominen, Elementary Goldstone Higgs boson and dark matter, Phys. Rev. D 91, 095021 (2015).

[9] E. Aprile et al. (XENON Collaboration), Observation of excess electronic recoil events in XENON1T, https:// doi.org/https://dx.doi.org/10.5281/zenodo.3924406.

[10] J. Wess and B. Zumino, Consequences of anomalous ward identities, Phys. Lett. 37B, 95 (1971).

[11] E. Witten, Global aspects of current algebra, Nucl. Phys. B223, 422 (1983).

[12] M. Frigerio, A. Pomarol, F. Riva, and A. Urbano, Composite scalar dark matter, J. High Energy Phys. 07 (2012) 015.

[13] Y. Wu, T. Ma, B. Zhang, and G. Cacciapaglia, Composite dark matter and Higgs, J. High Energy Phys. 11 (2017) 058.

[14] C. Cai, G. Cacciapaglia, and H.-H. Zhang, Vacuum alignment in a composite 2HDM, J. High Energy Phys. 01 (2019) 130.

[15] J. Galloway, J. A. Evans, M. A. Luty, and R. A. Tacchi, Minimal conformal technicolor and precision electroweak tests, J. High Energy Phys. 10 (2010) 086.
[16] T. Ma and G. Cacciapaglia, Fundamental composite 2HDM: SU(N) with 4 flavours, J. High Energy Phys. 03 (2016) 211.

[17] G. Cacciapaglia and F. Sannino, Fundamental composite (Goldstone) Higgs dynamics, J. High Energy Phys. 04 (2014) 111.

[18] J. Barnard, T. Gherghetta, and T. S. Ray, UV descriptions of composite Higgs models without elementary scalars, J. High Energy Phys. 02 (2014) 002.

[19] G. Ferretti and D. Karateev, Fermionic UV completions of composite Higgs models, J. High Energy Phys. 03 (2014) 077.

[20] L. Vecchi, A dangerous irrelevant UV-completion of the composite Higgs, J. High Energy Phys. 02 (2017) 094.

[21] G. Cacciapaglia, T. Ma, S. Vatani, and Y. Wu, Towards a fundamental safe theory of composite Higgs and dark matter, arXiv:1812.04005.

[22] C. Grojean, O. Matsedonskyi, and G. Panico, Light top partners and precision physics, J. High Energy Phys. 10 (2013) 160.

[23] D. Ghosh, M. Salvarezza, and F. Senia, Extending the analysis of electroweak precision constraints in composite Higgs models, Nucl. Phys. B914, 346 (2017).

[24] M. Bauer, M. Neubert, and A. Thamm, Collider probes of axion-like particles, J. High Energy Phys. 12 (2017) 044.

[25] M. Giannotti, I. G. Irastorza, J. Redondo, A. Ringwald, and K. Saikawa, Stellar recipes for axion hunters, J. Cosmol. Astropart. Phys. 10 (2017) 010.

[26] M. Giannotti, I. Irastorza, J. Redondo, and A. Ringwald, Cool WISPs for stellar cooling excesses, J. Cosmol. Astropart. Phys. 05 (2016) 057.

[27] G. Panico, M. Redi, A. Tesi, and A. Wulzer, On the tuning and the mass of the composite Higgs, J. High Energy Phys. 03 (2013) 051.

[28] E. Aprile et al. (XENON Collaboration), Physics reach of the XENON1T dark matter experiment, J. Cosmol. Astropart. Phys. 04 (2016) 027.

[29] B. Mount et al., LUX-ZEPLIN (LZ) technical design report, arXiv:1703.09144.

[30] B. Bhattacherjee and R. Sengupta, XENON1T excess: Some possible backgrounds, arXiv:2006.16172. 
[31] L. Di Luzio, M. Fedele, M. Giannotti, F. Mescia, and E. Nardi, Solar Axions Cannot Explain the XENON1T Excess, Phys. Rev. Lett. 125, 131804 (2020).

[32] A. Ayala, I. Domínguez, M. Giannotti, A. Mirizzi, and O. Straniero, Revisiting the Bound on Axion-Photon Coupling from Globular Clusters, Phys. Rev. Lett. 113, 191302 (2014).

[33] C. Gao, J. Liu, L.-T. Wang, X.-P. Wang, W. Xue, and Y.-M. Zhong, Re-Examining the Solar Axion Explanation for the XENON1T Excess, Phys. Rev. Lett. 125, 131806 (2020).

[34] C. Grojean and G. Servant, Gravitational waves from phase transitions at the electroweak scale and beyond, Phys. Rev. D 75, 043507 (2007).

[35] W.-C. Huang, F. Sannino, and Z.-W. Wang, Gravitational waves from Pati-Salam dynamics, arXiv:2004.02332.

[36] R. Akers et al. (OPAL Collaboration), Measurement of single photon production in e+ e- collisions near the Z0 resonance, Z. Phys. C 65, 47 (1995).

[37] M. Acciarri et al. (L3 Collaboration), Search for new physics in energetic single photon production in $e^{+} e^{-}$ annihilation at the $Z$ resonance, Phys. Lett. B 412, 201 (1997).

[38] P. Abreu et al. (DELPHI Collaboration), Search for new phenomena using single photon events in the DELPHI detector at LEP, Z. Phys. C 74, 577 (1997).

[39] J. Ahn et al. (KOTO Collaboration), Search for the $K_{L} \rightarrow$ $\pi^{0} \nu \bar{\nu}$ and $K_{L} \rightarrow \pi^{0} X^{0}$ Decays at the J-PARC KOTO Experiment, Phys. Rev. Lett. 122, 021802 (2019).

[40] A. J. Buras, D. Buttazzo, J. Girrbach-Noe, and R. Knegjens, $K^{+} \rightarrow \pi^{+} \nu \bar{\nu}$ and $K_{L} \rightarrow \pi^{0} \nu \bar{\nu}$ in the standard model: Status and perspectives, J. High Energy Phys. 11 (2015) 033.

[41] Y. Grossman and Y. Nir, $K_{L} \rightarrow \pi^{0}$ neutrino anti-neutrino beyond the standard model, Phys. Lett. B 398, 163 (1997).

[42] K. Fuyuto, W.-S. Hou, and M. Kohda, Loophole in $K \rightarrow \pi \nu \bar{\nu}$ Search and New Weak Leptonic Forces, Phys. Rev. Lett. 114, 171802 (2015).

[43] J. Lees et al. (BABAR Collaboration), Search for $B \rightarrow K^{(*)} \nu \bar{\nu}$ and invisible quarkonium decays, Phys. Rev. D 87, 112005 (2013).

[44] J. Grygier et al. (Belle Collaboration), Search for $\boldsymbol{B} \rightarrow \boldsymbol{h} \boldsymbol{\nu} \overline{\boldsymbol{\nu}}$ decays with semileptonic tagging at Belle, Phys. Rev. D 96, 091101 (2017).

[45] M. Tanabashi et al. (Particle Data Group), Review of Particle Physics, Phys. Rev. D 98, 030001 (2018).

[46] W. Altmannshofer, A. J. Buras, D. M. Straub, and M. Wick, New strategies for new physics search in $B \rightarrow K^{*} \nu \bar{\nu}$, $B \rightarrow K \nu \bar{\nu}$ and $B \rightarrow X_{s} \nu \bar{\nu}$ decays, J. High Energy Phys. 04 (2009) 022.

[47] A. J. Buras, J. Girrbach-Noe, C. Niehof, and D. M. Straub, $B \rightarrow K^{(*)} \nu \bar{\nu}$ decays in the standard model and beyond, J. High Energy Phys. 02 (2015) 184.

[48] C. Hambrock, A. Khodjamirian, and A. Rusov, Hadronic effects and observables in $B \rightarrow \pi \ell^{+} \ell^{-}$decay at large recoil, Phys. Rev. D 92, 074020 (2015).

[49] F. Takahashi, M. Yamada, and W. Yin, XENON1T anomaly from anomaly-free ALP dark matter and its implications for stellar cooling anomaly, arXiv:2006.10035.

[50] Y. Chen, J. Shu, X. Xue, G. Yuan, and Q. Yuan, Sun heated $\mathrm{MeV}$-scale dark matter and the XENON1T electron recoil excess, arXiv:2006.12447.
[51] H. An, M. Pospelov, J. Pradler, and A. Ritz, New limits on dark photons from solar emission and $\mathrm{keV}$ scale dark matter, arXiv:2006.13929.

[52] I. M. Bloch, A. Caputo, R. Essig, D. Redigolo, M. Sholapurkar, and T. Volansky, Exploring new physics with $\mathrm{O}(\mathrm{keV})$ electron recoils in direct detection experiments, arXiv:2006.14521.

[53] R. Budnik, H. Kim, O. Matsedonskyi, G. Perez, and Y. Soreq, Probing the relaxed relaxion and Higgs-portal with S1 \& S2, arXiv:2006.14568.

[54] C. Boehm, D. G. Cerdeno, M. Fairbairn, P. A. Machado, and A. C. Vincent, Light new physics in XENON1T, arXiv:2006 .11250 .

[55] A. Bally, S. Jana, and A. Trautner, Neutrino self-interactions and XENON1T electron recoil excess, arXiv:2006.11919.

[56] D. Aristizabal Sierra, V. De Romeri, L. Flores, and D. Papoulias, Light vector mediators facing XENON1T data, Phys. Lett. B 809, 135681 (2020).

[57] A. N. Khan, Can nonstandard neutrino interactions explain the XENON1T spectral excess?, Phys. Lett. B 809, 135782 (2020).

[58] Y. Jho, J.-C. Park, S. C. Park, and P.-Y. Tseng, Gauged lepton number and cosmic-ray boosted dark matter for the XENON1T excess, arXiv:2006.13910.

[59] S.-F. Ge, P. Pasquini, and J. Sheng, Solar neutrino scattering with electron into massive sterile neutrino, Phys. Lett. B 810, 135787 (2020).

[60] K. Kannike, M. Raidal, H. Veermäe, A. Strumia, and D. Teresi, Dark matter and the XENON1T electron recoil excess, arXiv:2006.10735.

[61] B. Fornal, P. Sandick, J. Shu, M. Su, and Y. Zhao, Boosted dark matter interpretation of the XENON1T excess, arXiv:2006.11264.

[62] K. Harigaya, Y. Nakai, and M. Suzuki, Inelastic dark matter electron scattering and the XENON1T excess, Phys. Lett. B 809, 135729 (2020).

[63] Q.-H. Cao, R. Ding, and Q.-F. Xiang, Exploring for sub$\mathrm{MeV}$ boosted dark matter from xenon electron direct detection, arXiv:2006.12767.

[64] R. Primulando, J. Julio, and P. Uttayarat, Collider constraints on a dark matter interpretation of the XENON1T excess, arXiv:2006.13161.

[65] H. M. Lee, Exothermic dark matter for XENON1T excess, arXiv:2006.13183.

[66] M. Baryakhtar, A. Berlin, H. Liu, and N. Weiner, Electromagnetic signals of inelastic dark matter scattering, arXiv: 2006.13918.

[67] J. Bramante and N. Song, Electric but not eclectic: Thermal relic dark matter for the XENON1T excess, arXiv:2006 .14089 .

[68] H. An and D. Yang, Direct detection of freeze-in inelastic dark matter, arXiv:2006.15672.

[69] L. Su, W. Wang, L. Wu, J. M. Yang, and B. Zhu, Xenon1T anomaly: Inelastic cosmic ray boosted dark matter, arXiv: 2006.11837.

[70] D. McKeen, M. Pospelov, and N. Raj, Hydrogen portal to exotic radioactivity, arXiv:2006.15140.

[71] G. Alonso-Álvarez, F. Ertas, J. Jaeckel, F. Kahlhoefer, and L. Thormaehlen, Hidden photon dark matter in the light of XENON1T and stellar cooling, arXiv:2006.11243. 
[72] G. Choi, M. Suzuki, and T. T. Yanagida, XENON1T Anomaly and its implication for decaying warm dark matter, arXiv:2006.12348.

[73] K. Nakayama and Y. Tang, Gravitational production of hidden photon dark matter in light of the XENON1T excess, arXiv:2006.13159.

[74] N. F. Bell, J. B. Dent, B. Dutta, S. Ghosh, J. Kumar, and J. L. Newstead, Explaining the XENON1T excess with luminous dark matter, arXiv:2006.12461.

[75] G. Paz, A. A. Petrov, M. Tammaro, and J. Zupan, Shining dark matter in Xenon1T, arXiv:2006.12462.

[76] U. K. Dey, T. N. Maity, and T. S. Ray, Prospects of Migdal effect in the explanation of XENON1T electron recoil excess, arXiv:2006.12529.
[77] L. Zu, G.-W. Yuan, L. Feng, and Y.-Z. Fan, Mirror dark matter and electronic recoil events in XENON1T, arXiv: 2006.14577.

[78] M. Lindner, Y. Mambrini, T. B. de Melo, and F. S. Queiroz, XENON1T anomaly: A light $Z^{\prime}$, arXiv:2006 .14590 .

[79] J. Smirnov and J. F. Beacom, Co-SIMP Miracle, Phys. Rev. Lett. 125, 131301 (2020).

[80] M. Cobal, C. De Dominicis, M. Fabbrichesi, E. Gabrielli, J. Magro, B. Mele, and G. Panizzo, Z-boson decays into an invisible dark photon at the LHC, HL-LHC and future lepton colliders, Phys. Rev. D 102, 035027 (2020). 